\title{
Engineering Design Contests and Courses: a Model-Based Taxonomy
}

\author{
Jason A. Foster \\ Senior Lecturer in Engineering Design Education, \\ Division of Engineering Science \\ University of Toronto \\ jason.foster@utoronto.ca
}

\author{
Patricia K. Sheridan \\ $\mathrm{PhD}$ Candidate \\ University of Toronto \\ patricia.sheridan@utoronto.ca
}

\begin{abstract}
Engineering faculty develop both design courses and design contests and competitions. These design experiences target and involve a wide variety of participants including students, faculty members, and members of the engineering profession. Given constraints on faculty time and resources, a common taxonomy and set of archetypes has the potential to increase the efficiency and effectiveness of the design of these experiences. Such a taxonomy can also prompt discussion among engineering design educators regarding their design pedagogy. This paper presents the initial development of such a taxonomy by modeling design experiences using an engineering design process model. Each stage of the design process model has been augmented with a set of common design decisions found in such experiences at the University of Toronto. Although preliminary, this augmented model shows promise as the foundation of both a taxonomy of design experiences and a handbook of design experience design.
\end{abstract}

\section{Introduction}

Engineering design experiences are proliferating across the Canadian educational landscape in a variety of both curricular and co-curricular forms. In university curricula, the Canadian Engineering Accreditation Board (CEAB) requires that every Canadian engineering program include at a minimum a capstone design course [1]. Many programs also include a cornerstone course and one or more intermediate design courses. Students, both undergraduate and in grade and high school, also have the opportunity to participate in many engineering design contests, competitions, and other co-curricular experiences. These co-curricular experiences are developed both by Faculties of Engineering and by external organizations, such as the Canadian Federation of Engineering Students, FIRST Robotics
Canada, and the James Dyson Foundation to name a few. While all of these curricular and co-curricular experiences focus on engineering design, they differ significantly in their goals and approaches, both within and across these groupings.

At the University of Toronto (UofT), design contests have as primary goals both outreach, usually to prospective high school students, and co-curricular enrichment, usually on behalf of the engineering profession or professional associations. Contest topics range from general or single-topic design for grade school and high school students, through to interdisciplinary undergraduate "Mega Projects" for upper year students. The deliverables required by these contests also vary immensely, ranging from (semi-)functional gadgets, to more abstract conceptual designs substantiated through research and simulation, through to fully functioning, production-ready products. A similar variety of goals, topics, and foci can be found in the design courses offered within the faculty at the undergraduate and graduate levels. Cornerstone, Capstone, and intermediate design courses vary in their relative emphasis on, at a minimum, the product, process, and learning that results from their constituent design activities.

Based on direct experience at the Universities of Toronto, Waterloo, and Western Ontario, in many cases the same individuals and groups are involved in developing engineering design experiences. These developers have to navigate the very different goals and contexts of these experiences, while maintaining integrity with their own beliefs about, and approaches to, engineering design. These challenges occur for both student organizers and faculty advisers when developing, administering, and judging design contests, and for faculty and teaching teams when developing and delivering integrated, comprehensive, engineering design curricula. These challenges are exacerbated when the objectives of the experience are poorly defined or communicated, or when the 
rationale behind the key elements of the experience is poorly understood.

In this paper we propose using an engineering design process model to frame a general engineering design experience, present an enumeration of common design decisions made when developing such experiences, and propose a small set of archetypes that can form the basis of specific engineering design experiences.

\section{Modeling the Design of Design Experiences}

Many engineering design courses present students with process (e.g. Waterfall, Spiral) or managerial (e.g. PERT, Gantt) models of design activities. Examples of both types of models can be found in many engineering design textbooks. While we teach students to use these models within the experiences we design, there is little evidence that we design, conceptualize, or present our engineering design experiences using these models.

Engineering design contests and courses can be seen as designed experiences intended to promote specific outcomes among the participants. These outcomes can range from an increased awareness of the engineering profession, to discipline-specific awareness, training in specific tools and techniques, through to having students develop their own philosophy on and approach to engineering design. Designing these experiences involves making specific decisions such as: who determines the particular design problem; how solutions are developed and analysed; and, how designs are assessed, judged, or selected. All of these decisions should support achieving the desired outcomes. The emerging field of "experience design" offers one approach to formalizing and structuring the design of experiences such as courses and contests.

We propose that a more traditional engineering design approach to the design of design experiences offers similar benefits. This approach has the advantage of being more familiar to engineering design practitioners. We also propose that an engineering design experience can be usefully modelled using the same process models used to model an engineering design activity. For example a specific engineering design experience (e.g. the Junior Design component of the University of Toronto Engineering Kompetition) can be modelled as a collection of decisions (e.g. predefined stakeholders, validation through experimentation) superimposed on a general design process. The result is a nested model in which the experience designer follows an engineering design process (the "development process" or DP) that results in a tailored engineering design process (the "experience process" or EP). The DP models the activities undertaken by the experience designer to create the experience; the EP models the activities that the participants (e.g. students or competitors) will be guided through during their design experience.

The remainder of this paper will focus on the EP rather than the DP. Doing so allows us to focus on the decisions available at each stage of the EP that allow it to meet the objectives of the DP, and on building a common taxonomy of design experiences.

Other ways of modeling an engineering design experience, for example using behaviourist or cognitivist models of learning, can provide additional insights ignored by a design process model. However we feel that the advantages gained by using a model familiar to engineering designers, and by using the same process model to describe both the act of designing an experience and the resulting experience itself, compensate for these omissions.

\section{Common Decisions in the Experience Process}

We have selected the engineering design process model developed by Roe, Soulis and Handa as the basis for the EP [2]. This process model was selected because of familiarity and because the 12 stages of the model provide a balance between generalizability and specificity. Given the isomorphic relationships among many engineering design process models, almost any model could be used to usefully describe and design an engineering design experience.

The sets of choices available at each stage of the experience process were developed by participating in and exploring a wide spectrum of the engineering design contests and courses that are required, or are available to students, at UofT. All of these experiences require participants to produce either a conceptual or detailed design whose viability needs to be demonstrated to a team of assessors.

The following subsections present a brief overview of, and the common choices available for, each stage of the engineering design process developed in [2] in the context of designing an engineering design experience.

\subsection{Defect or Lack in Environment}

Involves identifying the problem to be addressed by the experience participants. Common decisions in the context of a design experience include: 
- A single problem is assigned to the participants by the experience designer

- A set of problems is provided to the participants by the experience designer, and the participants can select from that set

- Participants are guided towards specific domains but no problems are given

- Participants identify their own problems with no restrictions on domain

- The experience design can specify that the problem be one of inter-, intra-, multi-, or transdisciplinary

\subsection{Need Analysis}

Involves identifying and determining the needs of stakeholders relevant to the problem being addressed. Common decisions in the context of a design experience include:

- Stakeholders are identified by the experience designer, and their needs elicited and codified prior to the experience

- Stakeholders are identified by the experience designer, and are brought to the experience so that participants can elicit their needs

- Stakeholders are identified by the participants, and their needs are elicited and codified during the experience by the experience designer

- Participants must both identify and solicit needs from stakeholders

- Need analysis can be scaffolded (e.g. through a template based on requirements engineering literature)

\subsection{Definition of the Design Problem}

Involves translating the general description of the design problem and stakeholder needs into an engineering framing. Common decisions in the context of a design experience include:

- The experience designer can specify the nature of the solution to the design problem (e.g. a device, process, or system)

- The experience designer can specify whether and the extent to which the problem context is explored and codified

- The engineering framing is provided by the experience designer
- Participants are provided with a skeleton that defines an engineering framing and map the problem and stakeholder needs to this framing

- Participants are provided with selected engineering resources (e.g. handbooks, textbooks, organization-specific guidelines) and are responsible for developing the engineering framing

- Participants develop the engineering framing

\subsection{Development of Design Criteria}

Involves developing engineering criteria (and implicitly constraints) used to assess potential solutions. A fully specified criterion comprises a metric, a gradient, and any boundary values. Common decisions include:

- The experience designer fully specifies a complete set of criteria

- The experience designer partially specifies a complete set of criteria, leaving the participants to determine one or more of boundary values, gradients, or metrics

- The experience designer partially specifies a partial set of criteria, leaving the participants both to expand and complete the set

- Participants develop the design criteria

- The experience designer can scaffold participant activities by specifying that criteria from one or more Design for X (DFx) fields be included

- The experience designer can require that nontraditional engineering requirements (e.g. nonfunctional, cultural, participant) be included

\subsection{Development of Alternative Solutions}

Involves developing a candidate set of alternatives that can be analyzed for feasibility using the criteria developed previously. Common decisions in the context of a design experience include:

- The experience designer specifies a minimum or maximum number of candidate solutions that participants must develop

- The experience designer specifies either specific idea generation tools that participants must use, or requires that a minimum number of such tools be used 


\subsection{Analysis of Feasibility}

Involves assessing the alternative solutions generated previously against the previously developed criteria. Common decisions in the context of a design experience include:

- The experience designer specifies a specific method or methods of analysis (e.g. experimentation, analytic calculation, reference designs, argument)

\subsection{Optimization}

Involves improving each feasible solution to the point where each member of the set of solutions has been developed to a similar degree. This process stage has been omitted from discussion as in our experience it is implied when iterating between developing alternative solutions and selecting the solution to be used.

\subsection{Selection of the Solution to be Used}

Involves selecting a single, preferred design solution from the set of feasible solutions. Common decisions in the context of a design experience include:

- The experience designer specifies a specific approach to comparing the results of the analysis across the alternative solutions (e.g. through a multi-criteria decision making (MCDM) tool such as Pugh Charts, Pairwise Comparison Matrices, the Analytic Hierarchy Process, etc.)

- Participants are responsible for selecting the preferred solution using a credible means of selection

\subsection{Implementation and Communication}

\subsubsection{Implementation}

Involves developing the final form of the design that will be provided or presented to stakeholders and assessors. In the context of design experiences, there are significant variations depending on the resources available to the participants. Common decisions in the context of a design experience include:

- Participants develop a conceptual design, possibly supported by drawings, models, prototypes, implementation plans, etc.

- Participants develop a functional or preproduction prototype, suitable for testing
- Participants develop a single-context implementation suitable for production use

- Assessment of the implementation is performed by all stakeholders

- Assessment of the implementation is performed by those facilitating the design experience, possibly with input from the remaining stakeholders

\subsubsection{Communication}

Involves communicating the design to stakeholders and assessors. Common decisions in the context of a design experience include:

- The experience designer specifies which communication modalities are required (e.g. poster, formal or informal, presentation, report)

- Assessment of the communication is performed by all stakeholders

- Assessment of the communication is performed by those facilitating the design experience, possibly with input from the remaining stakeholders

\subsection{Transfer to Detailed Phase}

Not applicable given the scope of the engineering design experiences considered for this paper.

\subsection{Rectified Defect or Lack in Environment}

Not applicable given the scope of the engineering design experiences considered for this paper.

\subsection{Information}

Refers to the type and breadth of information that the participants are expected to incorporate into their design experience. Common decisions in the context of a design experience include:

- The experience designer selects, summarizes, and provides a complete information package to the participants

- The experience designer selects core information for the participants, and provides them with references to this information

- The experience designer introduces the participants to appropriate information sources

- Participants are required to identify and locate any necessary information 


\section{Application of the Experience Process Model}

The model of the Experience Process presented previously can be used both descriptively and generatively. When used as a taxonomy to describe existing design experiences, the morphology provides a common basis and set of terms that enable more efficient and effective communication and understanding. The following section demonstrates how the model can be used to describe ESC102, a first year service design course.

\subsection{ESC102 Calendar Description}

A studio-based, service learning course in which students work in small teams to identify and then to design solutions for a contemporary issue situated within the Greater Toronto Area. The Design component of the course introduces formal design techniques such as framing, requirements gathering and codification, processes and heuristics, planning, and multi-criteria decision making. The Communication component introduces communicative genres such as Requests for Proposals (RFPs), brochures, posters, and oral presentations. Material from other concurrent courses is integrated through targeted activities and expectations in the Design and Communication components [3].

\subsection{ESC102 Framed using the Experience Process model}

\subsubsection{Defect or Lack in Environment}

- Provided by the course instructors as "Sustainably improve the City of Toronto"

\subsubsection{Need Analysis}

- Students must both identify and solicit needs from stakeholders

\subsubsection{Definition of the Design Problem}

- Students are responsible for defining the design problem

\subsubsection{Development of Design Criteria}

- Students are responsible for developing the design criteria
- Students must include "sustainability" as a criteria

- Students must include criteria drawn from at least three DFx literatures

- Instructors provide meta-level requirements (e.g. "Must be solvable in 6 weeks by a team of three, first year students")

\subsubsection{Development of Alternative Solutions}

- Students must develop multiple, credible design concepts

\subsubsection{Analysis of Feasibility}

- Students must demonstrate the feasibility of their design concepts through a credible engineering argument based on (e.g.) reference designs, calculations, prototypes and experimentation, etc.

\subsubsection{Selection of the Solution to the Used}

- Students must use at least one formal multicriteria decision making approach to make their selection

\subsubsection{Implementation and Communication - Implementation}

- Students must develop at least one prototype of their chosen design

- Students can choose the fidelity and medium of their prototype(s)

\subsubsection{Implementation and Communication - Communication}

- Students must present their design in a short presentation

- Students must develop a printed poster describing their design

\subsubsection{Information}

- Students are provided with an orientation to the UofT engineering library

\section{Generative Archetypes}

The morphology can be used generatively in two ways. The first way involves having the experience 
designer work through the model, selecting a specific choice at each stage. The second way has the designer start with an archetypal design experience which they then customize to suit their specific objectives and context. This second approach has the advantage of avoiding sets of choices that have not been validated through experience.

In developing the morphology of the experience model, we have inductively identified four archetypal engineering design experiences:

- Prefabricated STEM Outreach

- Co-curricular Application Specific

- Contract Research \& Development

- Personal Growth and Exploration

Due to space constraints, the specific decisions that make up each archetype have been omitted.

Additional archetypes will likely be identified and codified as we use this model to describe a wider variety of engineering design experiences.

\section{Conclusions and Future Work}

Using a design process model to describe many of the different design experiences offered at the University of Toronto has enabled us to better understand those experiences and to explore ways of improving the experiences that we have already designed. The structured description and common vocabulary has highlighted key differences in the approach used by different experience designers, and has prompted discussions among the designers regarding their engineering design pedagogy. It has also in one instance helped accelerate the formation of a competition judging team by suggesting questions to ask both within the team and between the judging team and the competition organizers. Given the early stages of this work, the early results are encouraging.

In the immediate term, future work on this taxonomy will focus on the following activities:

- reviewing a larger set of design experiences to expand the sets of known decisions

- identifying additional generative archetypes

- $\quad$ exploring the Development Process, in addition to the Experience Process, to better understand the experience objectives and the approaches taken by experience designers

- incorporating alternative models drawn from education and learning theory

The end goal of this work is to develop a design handbook that experience designers can use as they create and refine their engineering design experiences.

\section{References}

[1] Canadian Engineering Accreditation Board. "Accreditation Criteria and Procedures." Internet: http://www.engineerscanada.ca/e/files/Accreditation_C riteria_Procedures_2010.pdf, 2010 [May 6, 2011].

[2] P.H. Roe, G.N. Soulis, and V.K. Handa, the discipline of design, The University of Waterloo, Waterloo, ON, 1992, pp. 132.

[3] University of Toronto - Faculty of Applied Science and Engineering "Course Descriptions." Internet: http://www. apsc.utoronto.ca/Calendars/20112012/Course-Descriptions .html 2011 [May 6, 2011]. 